\title{
The anti-inflammatory and antifibrotic effects of Coreopsis tinctoria Nutt on high-glucose-fat diet and streptozotocin-induced diabetic renal damage in rats
}

\author{
Lan Yao', Linlin Li ${ }^{2}$, Xinxia Li ${ }^{3}$, Hui Li ${ }^{3}$, Yujie Zhang ${ }^{1}$, Rui Zhang ${ }^{2}$, Jian Wang ${ }^{2}$ and Xinmin Mao ${ }^{1 *}$
}

\begin{abstract}
Background: Diabetic nephropathy is a serious complication of diabetes whose development process is associated with inflammation, renal hypertrophy, and fibrosis. Coreopsis tinctoria Nutt, traditionally used as a healthcare tea, has anti-inflammatory, anti-hyperlipidemia, and glycemic regulation activities. The aim of our study was to investigate the renal protective effect of ethyl acetate extract of C. tinctoria Nutt (AC) on high-glucose-fat diet and streptozotocin (STZ)-induced diabetic rats.

Methods: A diabetic rat model was induced by high-glucose-fat diet and intraperitoneal injection of $35 \mathrm{mg} / \mathrm{kg}$ STZ. After treatment with AC at a daily dose of 150, 300 or, $600 \mathrm{mg} / \mathrm{kg}$ for 4 weeks, metabolic and renal function parameters of serum and urine were examined. Degree of renal damage, renal proinflammatory cytokines, and fibrotic protein expression were analyzed by histopathology and immunohistochemistry. Renal AMP-activated protein kinase (AMPK) and transforming growth factor (TGF)- $31 /$ Smad signaling pathway were determined by western blotting.

Results: Diabetic rats showed obvious renal dysfunction, inflammation and fibrosis. However, AC significantly reduced levels of blood glucose, total cholesterol, triglyceride, blood urea nitrogen, serum creatinine and urinary albumin, as well as expression of kidney proinflammatory cytokines of monocyte chemoattractant protein-1 and intercellular adhesion molecule-1. AC also ameliorated renal hypertrophy and fibrosis by reducing fibronectin and collagen IV and suppressing the TGF- $31 /$ Smad signaling pathway. Meanwhile, AMPKa as a protective cytokine was markedly stimulated by AC.
\end{abstract}

Conclusion: In summary, AC controls blood glucose, inhibits inflammatory and fibrotic processes, suppresses the TGF- $\beta 1 /$ Smad signaling pathway, and activates phosphorylation of AMPKa in the kidneys, which confirms the protective effects of AC in the early stage of diabetic kidney disease.

Keywords: Diabetic nephropathy, Coreopsis tinctoria Nutt, Anti-inflammatory effect, Anti-fibrotic effect

\section{Background}

As a result of socioeconomic development and lifestyle changes, prevalence of diabetes has risen rapidly in the past few decades. At present, diabetes has become a major public health problem in China, and solutions are urgently needed to prevent it as well as its complications $[1,2]$.

\footnotetext{
* Correspondence: mxm3277@sina.com

'College of Traditional Chinese Medicine, Xinjiang Medical University, Xinyi Street 393, Urumuqi 830011, China

Full list of author information is available at the end of the article
}

Diabetic nephropathy (DN) is one of the major complications of diabetes [3]. About $20 \%$ of patients with diabetes develop nephropathy after many years [4]. DN is becoming a high-incidence cause of end-stage renal disease (ESRD) in China and other countries $[5,6]$. Many studies suggest that uncontrolled hyperglycemia and hypertension may be risk factors for the development of DN [7]. Hypertrophy of glomerular structures, thickening of basement membranes, abnormal accumulation of extracellular matrix (ECM) proteins, including fibronectin and collagen, in the glomeruli, as well as

\section{() BioMed Central}

(c) 2015 Yao et al. Open Access This article is distributed under the terms of the Creative Commons Attribution 4.0 International License (http://creativecommons.org/licenses/by/4.0/), which permits unrestricted use, distribution, and reproduction in any medium, provided you give appropriate credit to the original author(s) and the source, provide a link to the Creative Commons license, and indicate if changes were made. The Creative Commons Public Domain Dedication waiver (http://creativecommons.org/publicdomain/zero/1.0/) applies to the data made available in this article, unless otherwise stated. 
persistent and slowly progressive proteinuria are the typical traits of DN [8-11].

Although much attention has been paid to strictly controlling blood glucose levels in diabetes, it is still difficult to prevent kidney disease [12, 13]. The pathogenesis of $\mathrm{DN}$ is complex and still not clear. However, recent experimental and clinical research has demonstrated that hyperglycemia induces renal oxidative stress, inflammation, and lipid accumulation, which leads to renal dysfunction by triggering multiple signaling pathways, and these may be crucial factors in pathogenesis of DN $[4,14,15]$.

Early diagnosis and preventive treatment is of major importance for reducing morbidity and mortality of DN. Although various interventions, such as angiotensinconverting enzyme inhibitors, angiotensin receptor blockers, vitamin D receptor agonist, or antihyperlipidemic agents are widely used, the rate of side effects and cardiovascular morbidity remains high [16]. Recently, traditional herbs have been used as effective complementary therapy in DN [17].

Coreopsis tinctoria Nutt is a plant of the genus Asteraceae in the family of Compositae. The native species is from North America, and then it spread worldwide. A large amount of $C$. tinctoria Nutt is distributed in South Xinjiang, China, where it is known as snow chrysanthemum [18]. The dry flowers of this plant are traditionally used as a healthcare tea to prevent cardiovascular disease and diabetes. Recent research has shown that extract of $C$. tinctoria Nutt has anti-inflammatory, anti-hyperlipidemia, and glycemic regulation activities [19-21]. Since these activities are closely related to metabolic regulation and diabetic kidney protection, we tried to identify the protective effect of ethyl acetate extract of $C$. tinctoria Nutt (AC) on diabetic kidney disease, and its possible mechanism of action.

\section{Methods}

Preparation of AC

C. tinctoria Nutt was harvested from Minfeng county, Hetian city in Xinjiang province, China. The species was identified by Professor Junping $\mathrm{Hu}$, College of Pharmacy, Xinjiang Medical University. The geographic coordinates of planted C. tinctoria Nutt was $82^{\circ}, 22^{\prime}, 00^{\prime \prime}-85^{\circ}, 55^{\prime}$, 00 " of east longitude and $35^{\circ}, 20 \mathrm{~min}, 00 \mathrm{~s}$ to $39^{\circ}$, $30 \mathrm{~min}, 00 \mathrm{~s}$ of north latitude. A voucher specimen of the plant material used in our study has been deposited in the herbarium of Ethnomedicine Research Institution in Urumuqi, Xinjiang province (No.20120715278). The dried flowers of C. tinctoria Nutt (160 g) were ground into powder and placed in $4 \mathrm{~L}$ of $55 \%$ ethanol for reflux extraction twice at $80{ }^{\circ} \mathrm{C}$ for $2 \mathrm{~h}$. The extracting liquid was filtered, merged, and then concentrated by rotary evaporator (R-210; Buchi, Essen, Germany) into 1 L liquid extract. An equal volume of AcOEt was added into the $1 \mathrm{~L}$ of liquid extract and than was concentrated and spray dried to obtain the powder of AC (4.4\%, w/w:dry flower). The powder of $\mathrm{AC}$ and metformin were weighed and dissolved in distilled water containing $1 \%(\mathrm{~g} / 100 \mathrm{~mL})$ sodium carboxymethyl cellulose and ultrasonicated for $4 \mathrm{~h}$ until the powder was completely dissolved.

\section{Analysis of AC by HPLC/MS}

AC was analyzed by HPLC (Waters 2690) with a diode-array detector (Waters 2487) scanning from 200-600 nm. Ethyl acetate extract was seperated by Shim-pack VP-ODS column $(150 \times 4.6 \mathrm{~mm}, 5 \mu \mathrm{m})$ with the optimum condition as described previously [20]. The mobile phase contained solution A of $0.5 \%$ formic acid and solution B of acetonitrile, with a column temperature of $35{ }^{\circ} \mathrm{C}$ and flow rate of $0.3 \mathrm{~mL} / \mathrm{min}$. The elution program was as follows: $95 \%-80 \%$ of solution A and $5 \%-20 \%$ of solution B during the first $1 \mathrm{~h}$; $80 \%-60 \%$ of solution A and $20 \%-40 \%$ of solution $\mathrm{B}$ for the remaining $50 \mathrm{~min}$. A mass spectrometer (LCQ-DecA XP MAX Thermo) with a negative electrospray ionization mode was used. Mass spectrometer conditions were maitainted with a source temperature of $100{ }^{\circ} \mathrm{C}$, desolvation temperature of $350{ }^{\circ} \mathrm{C}$, desolvation gas flow of $600 \mathrm{~L} / \mathrm{h}$, capillary voltage of $3 \mathrm{kV}$, and cone voltage of $30 \mathrm{~V}$. Mass scan range was from 100 to $800 \mathrm{~m} / \mathrm{z}$.

\section{Animal models}

Healthy male Sprague-Dawley rats (8-10 weeks old, weighing 200-250 g) were obtained from the Animal Centre, Xinjinag Medical University (No. of Certification: 65000700000045). The study was authorized by the Institutional Animal Care and Use Committee of First Affiliated Hoapital of Xinjiang Medical University (Approval No.: IACUC-20140304011). The animals were kept in a specific pathogen free environment at $22 \pm 2{ }^{\circ} \mathrm{C}$ and $45 \pm 5 \%$ humidity for 2 weeks of acclimatization. Then, they were randomly divided into two groups and fed with normal diet and highfat diet (67 \% normal diet, $20 \%$ sucrose, $10 \%$ fatty oil, $2.5 \%$ cholesterol, and $0.8 \%$ sodium cholate). After 8 weeks feeding, the rats were fasted overnight and injected intraperitoneally with $35 \mathrm{mg} / \mathrm{kg}$ streptozotocin (STZ) dissolved in $0.1 \mathrm{M}$ citric acid and $0.1 \mathrm{M}$ sodium phosphate solution, $\mathrm{pH}$ 4.5. The diabetic model was confirmed after $72 \mathrm{~h}$ by a glucose level $\geq 16.7 \mathrm{mmol} / \mathrm{L}$. Animals were fed with high-fat diet until the end of the experiment.

\section{Experimental design}

Animals were randomly divided into six groups of ten according to the weight and blood glucose levels as follows: Group 1 (NC): normal control rats with distilled 
water containing $1 \%(\mathrm{~g} / 100 \mathrm{~mL})$ sodium carboxymethyl cellulose per day by gavage administration for 4 weeks; Group 2 (DC): diabetic control rats with distilled water containing $1 \%(\mathrm{~g} / 100 \mathrm{~mL})$ sodium carboxymethyl cellulose per day by gavage administration for 4 weeks; Groups 3, 4 and 5 (DC + AC150, DC + AC300 and DC + AC600): diabetic rats with 150,300 and $600 \mathrm{mg} / \mathrm{kg}$ AC per day, respectively, by gavage administration for 4 weeks; Group $6(\mathrm{DC}+\mathrm{M})$ : diabetic rats with $200 \mathrm{mg} / \mathrm{kg}$ metformin per day by gavage administration for 4 weeks.

At day 27 of adminstration, $24 \mathrm{~h}$ urine samples from rats housed in metabolic cages were centrifuged and collected. At the end of the experiments, all the animals were killed by $10 \%$ chloral hydrate. All the blood samples were collected. The right kidneys were rinsed with cold isotonic saline and placed immediately in liquid nitrogen, and then stored at $-80{ }^{\circ} \mathrm{C}$ for testing. The left kidneys were weighed and fixed in $10 \%$ formaldehyde solution.

\section{Blood sampling and analysis}

The collected blood samples were centrifuged at $1000 \mathrm{~g}$ for $20 \mathrm{~min}$. Serum samples were separated for detection of blood glucose, total cholesterol (TC), triglycerides, blood urea nitrogen (BUN), and serum creatinine $(\mathrm{SCr})$ by an automatic biochemical analyzer (BS-120; Mindray, China).

\section{Urinalysis}

Urine albumin levels were measured using a kit obtained from Nanjing Jiancheng Bioengineering Institute, China.

\section{Histopathological analysis}

Left kidney tissues were fixed in $10 \%$ formaldehyde solution, embedded in paraffin, and cut into $4 \mu \mathrm{m}$ sections. The tissue sections were stained with hematoxylin and eosin (H\&E), and Masson's reagent. Images were captured by a light microscope (SZX7-1093; Olympus, Japan). The mesangial expansion index from 30 glomeruli of each rat was graded in four levels from 0 to 3 [22]: 0, normal glomerulus; 1 , matrix expansion occurred in up to $50 \%$ of a glomerulus; 2, matrix expansion occurred in $50 \%-75 \%$ of a glomerulus; and 3, matrix expansion occurred in $75 \%-100 \%$ of a glomerulus, and the means were caculated and compared. For Masson's stain, $4^{\circ}$ of fibrosis from 30 glomeruli of each rat were scored by measuring the percentage area of blue staining as follows: 0, absent or < $25 \%$; 1, $25 \%-50 \%$; 2 , $50 \%-75 \%$; and $3,>75 \%$. The slides from all the animals were evaluated by a pathologist who was unaware of the experimental details.

\section{Immunohistochemical analysis}

After deparaffinization and hydration, the paraffin embedded tissue sections were washed three times with phosphate-buffered saline (PBS). Endogenous peroxidase activity was quenched by incubating the slides in methanol solution containing $3 \% \mathrm{H}_{2} \mathrm{O}_{2}$. After washing three times in distilled water and PBS, goat serum (Bioss Biotechnology, Beijing, China) was used to block the nonspecific binding sites for $30 \mathrm{~min}$. The primary antibodies [TGF- $\beta 1,1: 500$; monocyte chemoattractant protein (MCP)-1, 1:500; intercellular adhesion molecule (ICAM)-1, 1:500; fibronectin, 1:1000; Collagen IV, 1:1000; Abcam, Cambridge, MA, USA) were incubated and reacted at $4{ }^{\circ} \mathrm{C}$ overnight. On the next day, the secondary horseradish-peroxidase-conjugated antibody (Bioss Biotechnology) was added and reacted for $30 \mathrm{~min}$ at $37{ }^{\circ} \mathrm{C}$. After washing three times with PBS, tissue sections were immunostained with diaminobenzidine tetrahydrochloride as the substrate and hematoxylin as the counterstain. Negative control slices were made in the same way except for the first antibody. Four degrees of TGF- $\beta 1$, MCP-1, ICAM-1, Collagen IV and fibronectin expression in 30 glomeruli from each rat were graded by measuring the percentage area of positive staining as follows: 0 , absent or $<25 \% ; 1,25 \%-50 \% ; 2,50 \%-75 \%$; and $3,>75 \%$. The slides from all the animals were evaluated by a pathologist who was unaware of the experimental details.

\section{Western blot analysis}

The right kidneys were extracted with RIPA lysis buffer (Thermo, USA) containing a protease and phosphatase inhibitor cocktail to obtain the protein lysate. The concentration of the protein lysate was measured by BCA Protein Assay Kit (Thermo). Protein lysate $(20 \mu \mathrm{g})$ from each sample was separated by $12 \%$ SDS-PAGE at $80 \mathrm{~V}$ and transferred onto PVDF membrane (Millpore, USA). After blocking in $5 \%(\mathrm{v} / \mathrm{v})$ skimmed milk and Tris-buffered saline Tween (TBST) for $1 \mathrm{~h}$ and washing three times in TBST, the transferred membranes were incubated with the primary antibodies of TGF- $\beta 1, \quad \beta$-actin (1:1000; Abcam); Smad2, p-Smad2, AMP-activated protein kinase (AMPK) $\alpha, \mathrm{p}-\mathrm{AMPK} \alpha$, and GAPDH (1:1000; Cell Signaling Technology, Danvers, MA, USA) at $4{ }^{\circ} \mathrm{C}$ overnight. The probed membranes were washed three times in TBST and combined with alkaline-phosphataseconjuated secondary antibody (Invitrogen, Carlsbad, CA, USA) for $1 \mathrm{~h}$ at $37^{\circ} \mathrm{C}$. The protein band was colored by BCIP/NBT Substrate Kit (Invitrogen) and the band density was scanned and calculated by Quantity One v4.62 software. 
A

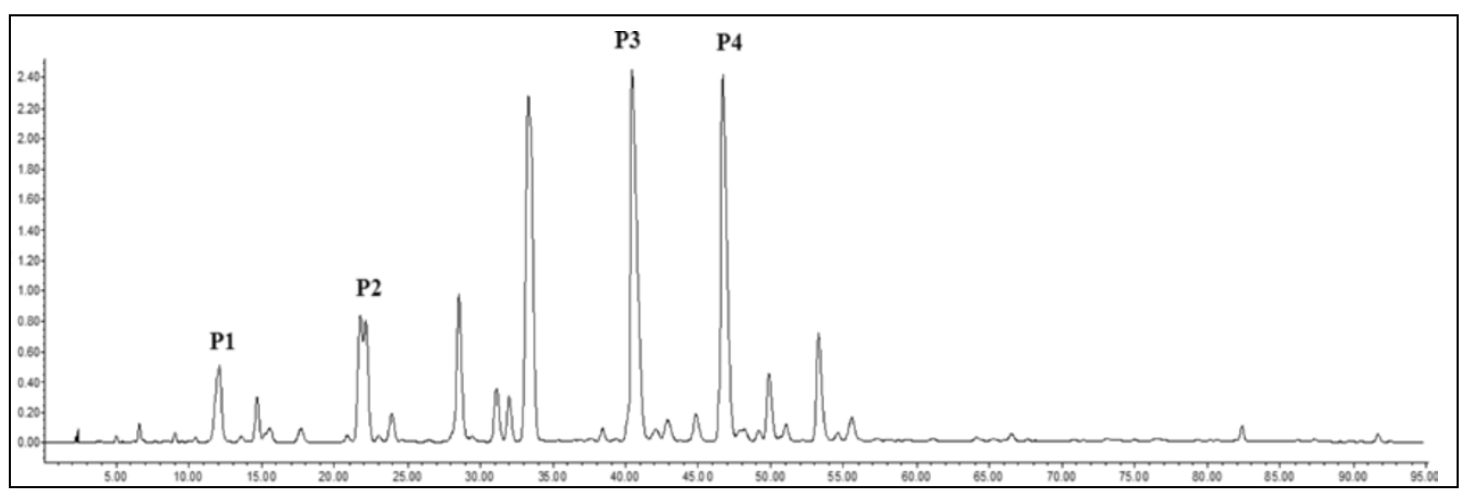

B

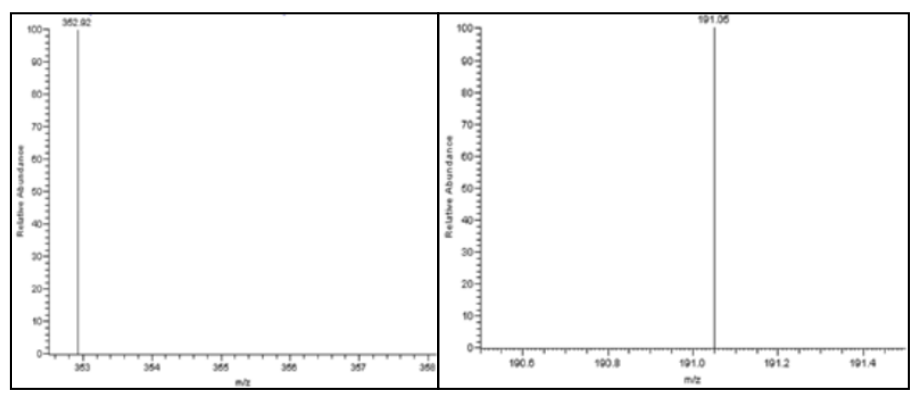

$\mathrm{C}$

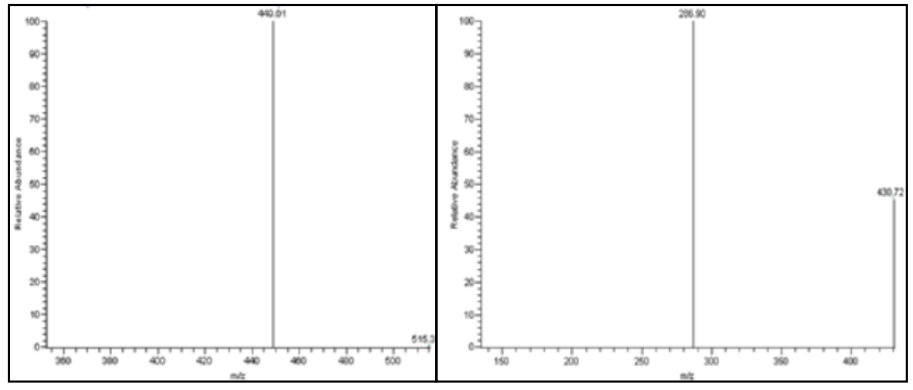

$\mathrm{D}$

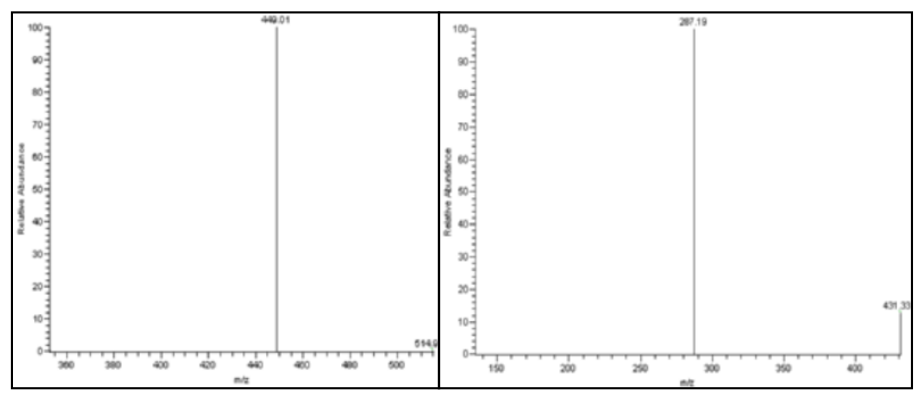

$\mathrm{E}$

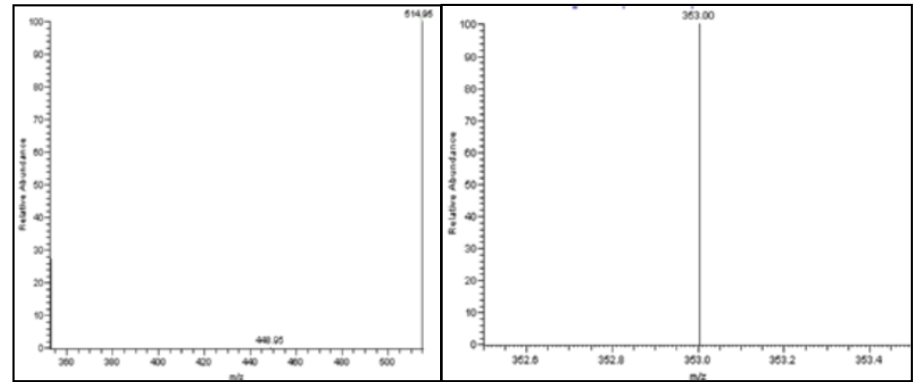

Fig. 1 HPLC/MS analysis of AC. a HPLC analysis of AC at $280 \mathrm{~nm}$ wavelength. Four main compounds were (P1) chlorogenic acid, (P2) flavanomarein, (P3) marein, and (P4) 4,5-dicaffeoyl-quinic acid. Precursor ion (left) and product ion (right) of (b) chlorogenic acid, (c) flavanomarein, (d) marein, and (e) 4,5-dicaffeoyl-quinic acid 


\section{Statistical analysis}

All data are presented as the mean \pm SEM. Differences between multiple groups were analysed by one-way analysis of variance followed by Duncan's multiple range test. Differences between two groups were measured by Student's $t$ test using SPSS v16.0 software (Chicago, IL, USA). Data were considerd statistically significant at $P<0.05$.

\section{Results}

\section{HPLC/MS analysis of AC}

The main compounds and their relative content were analyzed by HPLC/MS. As shown in Fig. 1 and Table 1, we identified chlorogenic acid, flavanomarein, marien, and 4,5-dicaffeoyl-quinic acid and determined their relative content.

\section{Change in body weight and metabolic parameters after treatment with $\mathrm{AC}$}

Diabetes was defined as blood glucose level $\geq 16.7 \mathrm{mmol} / \mathrm{L}$ in a random blood glucose test. The DC group had a significantly higher average random blood glucose level of $30.05 \mathrm{mmol} / \mathrm{L}$ (Table 2). However, after 4 weeks of treatment, the random blood glucose level in the positive control group $(P<0.05)$ and the low-dose AC group $(P<0.01)$ was significantly decreased. By contrast, body weight and serum insulin of the DC group were significantly decreased compared with those in the normal group. Treatment with $150 \mathrm{mg} / \mathrm{kg}$ AC alleviated the body weight $(P<0.05)$ and $600 \mathrm{mg} / \mathrm{kg} \mathrm{AC}(P<0.05)$ alleviated serum insulin loss. It was noteworthy that dyslipidemia appeared in the DC, DC + AC150 and $\mathrm{DC}+\mathrm{AC600}$ groups. The later two groups were found to ameliorate abnormity of TG and TC.

\section{Effect of $A C$ on renal function}

Kidney/weight ratio $(\mathrm{K} / \mathrm{W})$ of diabetic rats was markedly higher than in normal rats (Table 3 ). K/W only decreased significantly in the DC + AC600 group $(P<0.05)$. Twenty-four-hour urine volume was higher in diabetic than normal rats. There was no significant change in 24 h urine volume apart from in the $\mathrm{DC}+\mathrm{AC} 150$ group $(P<0.05)$. In diabetic rats, mean BUN, SCr and albuminuria were notably increased in comparison with normal rats. Compared with diabetic rats, BUN level in the DC + AC300, DC + AC600 and DC + M groups was significantly decreased $(P<0.05)$. SCr level in all the treatment groups was significantly decreased, with a greater decrease in the $\mathrm{DC}+150$ group $(P<0.01)$. Albuminuria was significantly ameliorated in the DC + AC600 group $(P<0.01)$.

\section{Effect of AC on renal histology}

H\&E and Masson's stain were used to detect renal histology characteristics. Judging from H\&E staining, glomerular and tubular lesions in the normal group were not obvious. The DC group showed a dramatic increase in mesangial matrix and basement membrane thickening, both in glomerular and tubular lesions (Fig. 2a). There was some improvement after treatment with $150 \mathrm{mg} / \mathrm{kg} \mathrm{AC}$ $(P<0.05), 300 \mathrm{mg} / \mathrm{kg} \mathrm{AC}(P<0.05), 600 \mathrm{mg} / \mathrm{kg} \mathrm{AC} \quad(P<$ $0.01)$ and $200 \mathrm{mg} / \mathrm{kg}$ metformin $(P<0.05)$. Collagen deposition was determined by Masson's stain. Heavy collagen deposition was clearly observed in the DC group. This abnormality was ameliorated after treatment with $\mathrm{AC}$ $(150 \mathrm{mg} / \mathrm{kg}, P<0.05 ; 300 \mathrm{mg} / \mathrm{kg}, P<0.05$; and $600 \mathrm{mg} / \mathrm{kg}$, $P<0.01)$ and metformin $(200 \mathrm{mg} / \mathrm{kg}, P<0.01)$. These results confirmed the protective effect of $\mathrm{AC}$ and metformin on diabetic renal damage.

\section{Effect of AC on renal TGF- $\beta 1$, MCP-1, ICAM-1, collagen IV and fibronectin expression}

To investigate the anti-inflammatory and anti-fibrotic effects of AC, immunohistochemistry was used to determine protein expression of TGF- $\beta 1$, MCP-1, ICAM-1, collagen IV and fibronectin in diabetic kidney. Positive expression was stained brown-yellow (Figs. 3a and 4a). Compared with the NC group, the DC group showed significant overexpression of the five cytokines (Figs. 3b and $4 \mathrm{~b} ; P<0.01)$. All cytokine expression was decreased to some extent after AC and metformin treatment. AC $600 \mathrm{mg} / \mathrm{kg}$ had the most inhibitory effect of protein expression of TGF- $\beta 1$, MCP-1, ICAM- 1 , collagen IV and fibronectin. These results indicate that $\mathrm{AC}$ has a protective effect on diabetic renal inflammation and fibrosis.

\section{AC ameliorates renal inflammation and fibrosis by activing AMPK and suppressing TGF- $\beta 1 /$ Smad signaling pathway}

To elucidate the mechanism of the anti-inflammatory and antifibrotic effects of $\mathrm{AC}$, the TGF- $\beta 1 / \mathrm{Smad}$ and AMPK signaling pathways were investigated by western blotting. The TGF- $\beta 1 /$ Smad signaling pathway was

Table 1 Analysis of relative quantity and compound identification of AC

\begin{tabular}{llcclll}
\hline Peak no. & Compund identification & Rt $(\mathrm{min})$ & Relative content $(\mathrm{mg} / \mathrm{g})$ & Precursor ion $(\mathrm{m} / \mathrm{z})$ & Product ion $(\mathrm{m} / \mathrm{z})$ & Molecular weight \\
\hline P1 & Chlorogenic acid & 17.76 & 4.08 & 354 & 353 & 191 \\
P2 & Flavanomarein & 26.3 & 34.73 & 450 & 449 & 431,287 \\
P3 & Marien & 46.55 & 181.61 & 450 & 449 & 431,287 \\
P4 & 4,5-dicaffeoyl-quinic acid & 52.66 & 100.51 & 516 & 515 & 353 \\
\hline
\end{tabular}


Table 2 Changes in body weight and metabolic parametres in each group

\begin{tabular}{llllll}
\hline Group & Body weigh $(\mathrm{g})$ & Plasma glucose $(\mathrm{mmo} / \mathrm{L})$ & $\mathrm{TC}(\mathrm{mmo} / \mathrm{L})$ & $\mathrm{TG}(\mathrm{mmol} / \mathrm{L})$ & Serum insulin $(\mu \mathrm{U} / \mathrm{mL})$ \\
\hline NC $(n=10)$ & $584.80 \pm 13.76$ & $7.10 \pm 0.12$ & $1.42 \pm 0.11$ & $1.58 \pm 0.07$ & $61.23 \pm 2.51$ \\
DC $(n=8)$ & $344.25 \pm 15.22 \#$ & $30.05 \pm 0.62 \#$ & $7.58 \pm 0.23 \#$ & $23.78 \pm 1.79 \#$ & $26.25 \pm 1.85 \#$ \\
DC + AC150 $(n=10)$ & $390.80 \pm 10.88^{*}$ & $21.67 \pm 1.79^{* *}$ & $2.11 \pm 0.33^{* *}$ & $7.09 \pm 1.17^{* *}$ & $23.25 \pm 1.47$ \\
DC + AC300 $(n=7)$ & $386.86 \pm 17.62$ & $30.9 \pm 0.55$ & $7.34 \pm 0.26$ & $20.27 \pm 2.9$ & $23.86 \pm 1.30$ \\
DC + AC600 $(n=9)$ & $374.22 \pm 12.25$ & $29.1 \pm 0.50$ & $4.23 \pm 0.26^{*}$ & $18.48 \pm 1.53^{*}$ & $31.91 \pm 1.34^{*}$ \\
DC + M $(n=10)$ & $379.1 \pm 11.49$ & $27.31 \pm 0.68^{*}$ & $4.31 \pm 0.20^{* *}$ & $17.22 \pm 0.90^{*}$ & $22.62 \pm 1.00$ \\
\hline
\end{tabular}

Data expressed as mean \pm SEM $(n=7-10)$. $\# P<0.01$ versus normal group; ${ }^{*} P<0.05$ versus control group; ${ }^{* *} P<0.01$ versus control group

considered to play a vital role in progression of renal fibrosis. As shown in Fig. 5a, b, TGF- $\beta 1$ expression in diabetic rats was significantly increased, followed by overexpression of phospho (p)-Smad2. Whereas, TGF- $\beta 1$ and $\mathrm{p}$-Smad2 expression was markedly decreased by treatment with AC. The active form of AMPK $\alpha$ was more highly expressed in normal rats than diabetic rats $(P<0.01)$. Treatment with AC $(150,300$ and $600 \mathrm{mg} / \mathrm{kg})$ and metformin significantly upregulated the p-AMPK $\alpha$ expression (Fig. 6a, b). These results indicated that the anti-inflammatory and antifibrotic effects of AC were possibly related to TGF- $\beta 1 / \mathrm{Smad}$ and AMPK signaling pathways.

\section{Discussion}

In present study, we demonstrated the renal protective effect of AC in diabetic rats. After treatment with AC for 4 weeks, both glucose and lipid metabolism disorders were ameliorated. Biochemical markers of renal function, such as albuminuria, BUN, SCr, K/W ratio and $24 \mathrm{~h}$ urine volume, as well as renal structural abnormalities and fibrosis, were significantly improved by AC treatment. We also demonstrated that $\mathrm{AC}$ inhibited renal inflammatory and fibrotic reactions and upregulated p-AMPK $\alpha$ expression, which suggests that the positive effects of $A C$ in diabetic nephropathy are multifactorial.

The animal model of high-fat diet combined with STZ-induced diabetes manifests many characteristics of human diabetes, such as hyperglycemia, hyperlipemia, lack of insulin secretion, and loss of body weight. Furthermore, kidney disease develops under these conditions. Compared with normal rats, diabetic rats showed significant increases in albuminuria, $\mathrm{BUN}, \mathrm{SCr}, \mathrm{K} / \mathrm{W}$ ratio and $24 \mathrm{~h}$ urine volume, along with renal inflammation and renal structural abnormalities and fibrosis, which indicated that the diabetic renal injury model was successfully formed.

Previous studies that demonstrated the anti-hyperlipemia and glycemic regulation activities of C. tinctoria Nutt $[19,20]$ were confirmed by our results. After treatment with low-and high-dose AC for 4 weeks, TC and triglyceride levels were significantly decreased. Blood glucose was only decreased in the low-dose AC group, and insulin secretion was only increased in the high-dose $\mathrm{AC}$ and metformin groups. It was surprising that the middle dose of AC had no effect on body weight and metabolic parameters, even when it ameliorated renal effects. The low dose of $\mathrm{AC}$ had a better effect on lipid regulation than the high dose had, even if there was less renal damage than with high dose AC. Further research is needed to reveal the relationship bewteen different doses of $\mathrm{AC}$ and their renal protective effect. Renal function parameters were improved by all three doses of AC. These results indicate that the protective effect of $\mathrm{AC}$ on $\mathrm{DN}$ is not only mediated by glycemic regulation, but also by multiple synergistic effects.

Inflammation has emerged as an important factor in understanding the pathogenic mechanism of DN. Activation of the inflammatory response, leading to upregulation of various inflammatory cytokines, chemokines, growth factors, adhesion molecules, and nuclear factors, is a common pathway for progression of $\mathrm{DN}$ in humans and in animal models $[14,15]$. MCP-1 is an important

Table 3 Changes in parametres of renal function in each group

\begin{tabular}{llllll}
\hline Group & K/W $(\mathrm{mg} / \mathrm{g})$ & $24 \mathrm{~h}$ urine volume $(\mathrm{mL} /$ day $)$ & $\mathrm{BUN}(\mathrm{mmo} / \mathrm{L})$ & $\mathrm{SCr}(\mu \mathrm{mol} / \mathrm{L})$ & Urine albumin $(\mathrm{mg} / 24 \mathrm{~h})$ \\
\hline $\mathrm{NC}(n=10)$ & $2.80 \pm 0.07$ & $18.5 \pm 1.85$ & $6.26 \pm 0.14$ & $102.53 \pm 0.86$ & $4.95 \pm 0.81$ \\
$\mathrm{DC}(n=8)$ & $5.15 \pm 0.10 \#$ & $157.88 \pm 4.56 \#$ & $15.96 \pm 0.93 \#$ & $128.56 \pm 6.08 \#$ & $34.59 \pm 2.42 \#$ \\
$\mathrm{DC}+\mathrm{AC} 150(n=10)$ & $4.80 \pm 0.17$ & $133.89 \pm 9.35^{*}$ & $13.05 \pm 1.14$ & $94.63 \pm 2.20^{* *}$ & $30.73 \pm 1.53$ \\
$\mathrm{DC}+\mathrm{AC} 300(n=7)$ & $5.01 \pm 0.27$ & $142.86 \pm 8.08$ & $11.59 \pm 1.5^{*}$ & $118.43 \pm 4.98^{*}$ & $28.40 \pm 1.44^{*}$ \\
$\mathrm{DC}+\mathrm{AC600}(n=9)$ & $4.71 \pm 0.16^{*}$ & $144.50 \pm 7.54$ & $13.37 \pm 0.70^{*}$ & $105.60 \pm 4.59^{*}$ & $26.75 \pm 2.32^{* *}$ \\
$\mathrm{DC}+\mathrm{M}(n=10)$ & $5.03 \pm 0.17$ & $143.70 \pm 5.97$ & $12.52 \pm 1.13^{*}$ & $103.04 \pm 3.46^{*}$ & $27.89 \pm 1.74^{*}$ \\
\hline
\end{tabular}

Data expressed as mean \pm SEM $(n=7-10)$. $\# P<0.01$ versus normal group; ${ }^{*} P<0.05$ versus control group; ${ }^{* *} P<0.01$ versus control group 


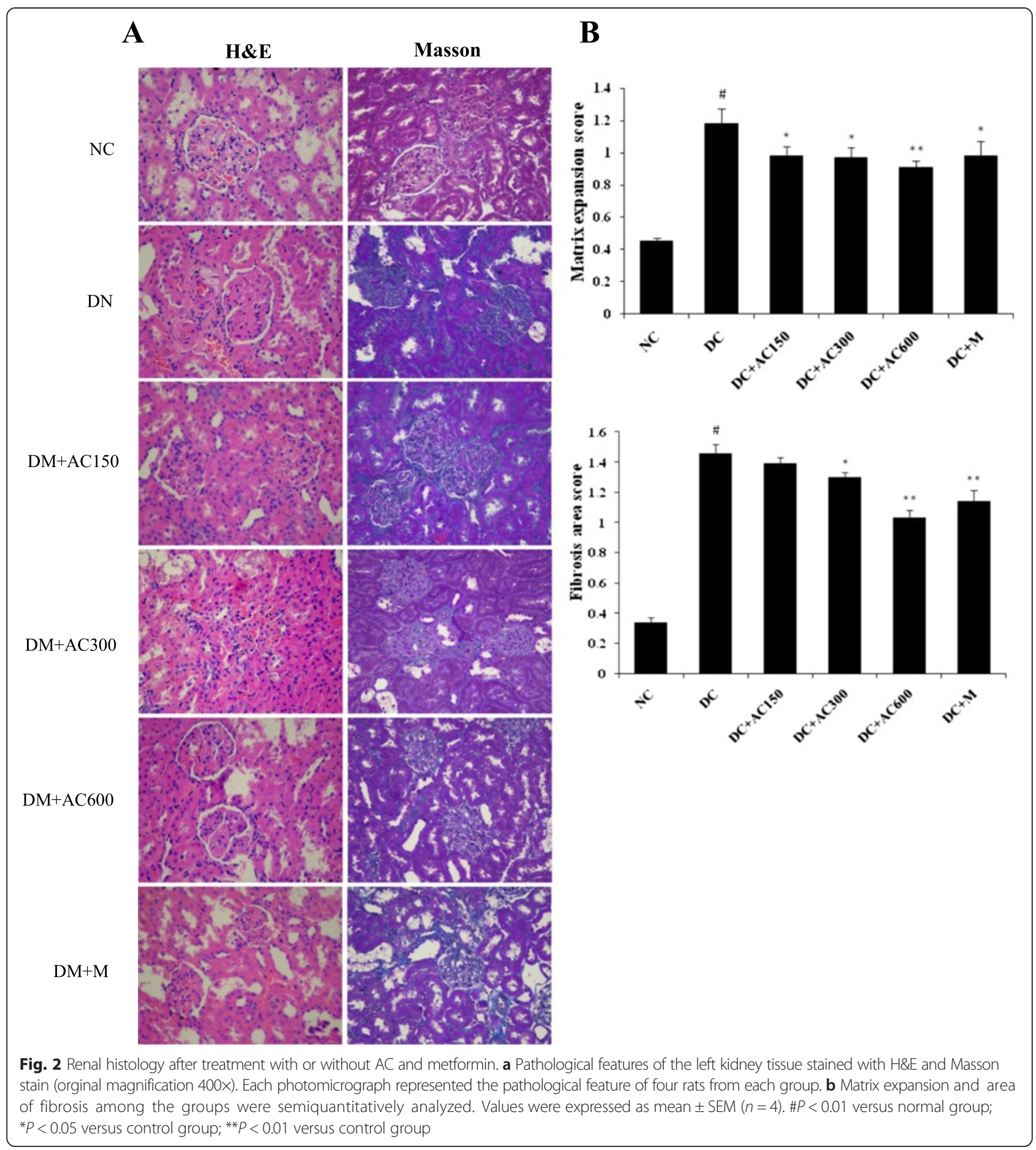

inflammatory cytokine that takes part in the development of DN. Evidence demonstrates that metabolic disorders induced by diabetes could drive MCP-1 activation and accelerate macrophage recruitment from the circulation to the kidneys. Meanwhile, high expression of MCP-1 aggravates ECM accumulation and protein excretion in glomeruli, tubules and interstitium, by activating overexpression of other proinflammatory cytokines [23, 24]. Also, MCP-1 can modulate mesangial matrix accumulation by TGF- $\beta 1$ production without affecting glomerular macrophage accumulation [25]. ICAM-1 is a cell surface glycoprotein that is expressed on kidney endothelial, epithelial and mesangial cells and leukocytes. ICAM-1 promotes macrophage infiltration 


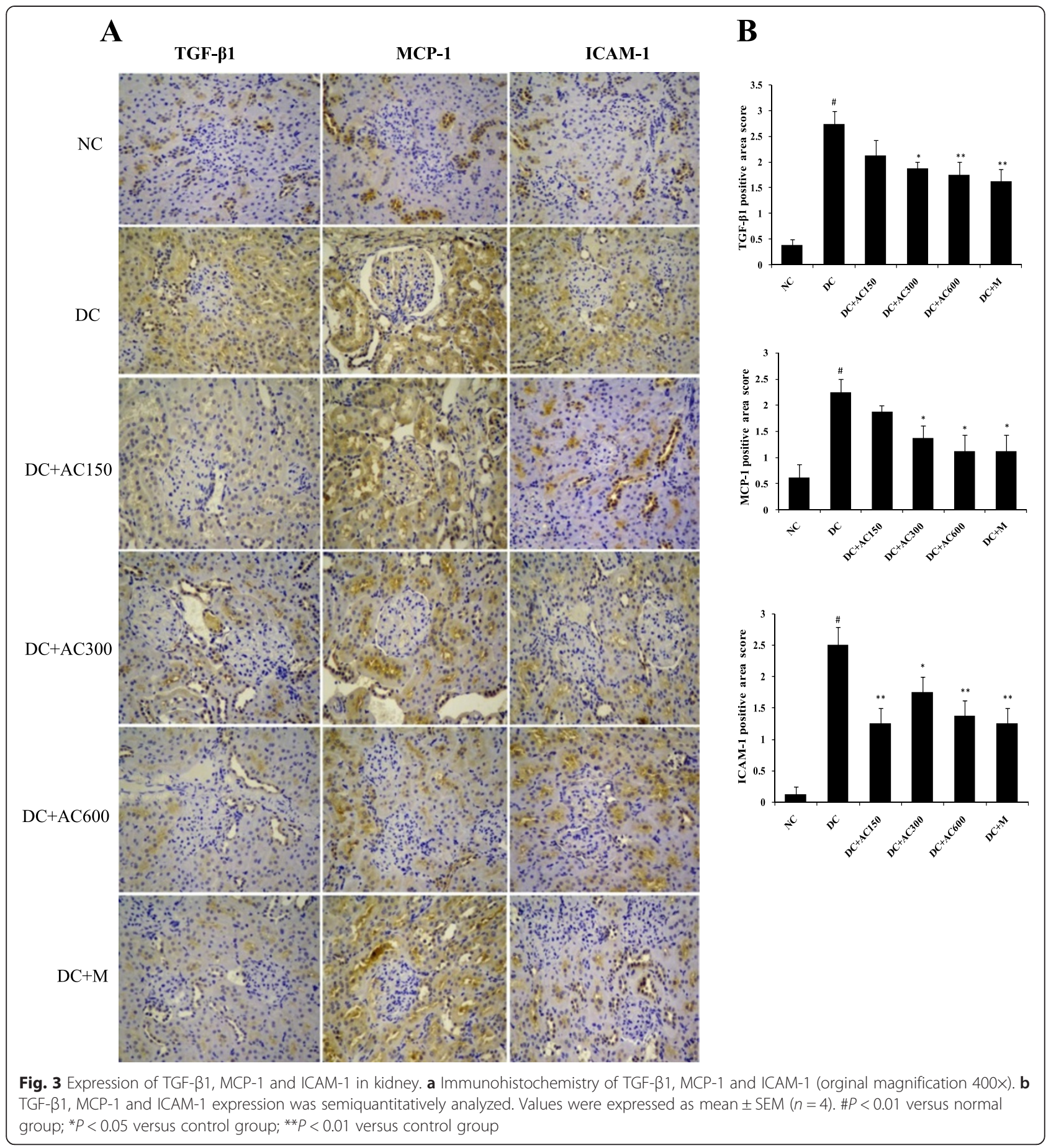

in glomeruli and interstitium, which is thought to play a crucial role in renal injury and proteinuria. Although the precise role of ICAM-1 in the development of DN has not been fully investigated, accumulating evidence has confirmed a close relationship. Proteinuria increases with overexpression of ICAM-1, which indicates that ICAM-1 could be a useful biomarker for prediction of DN [26]. Consistent with these studies, we found that both MCP-1 and ICAM-1 expression was markedly increased in rat diabetic kidneys, While AC treatment dramatically decreased both MCP-1 and ICAM-1 expression. These results indicate the renal improvement effect of AC is possibly mediated by its anti-inflammatory activity.

A large body of evidence shows that TGF- $\beta 1$ is a key participant in the cascade of events of kidney sclerosis. 


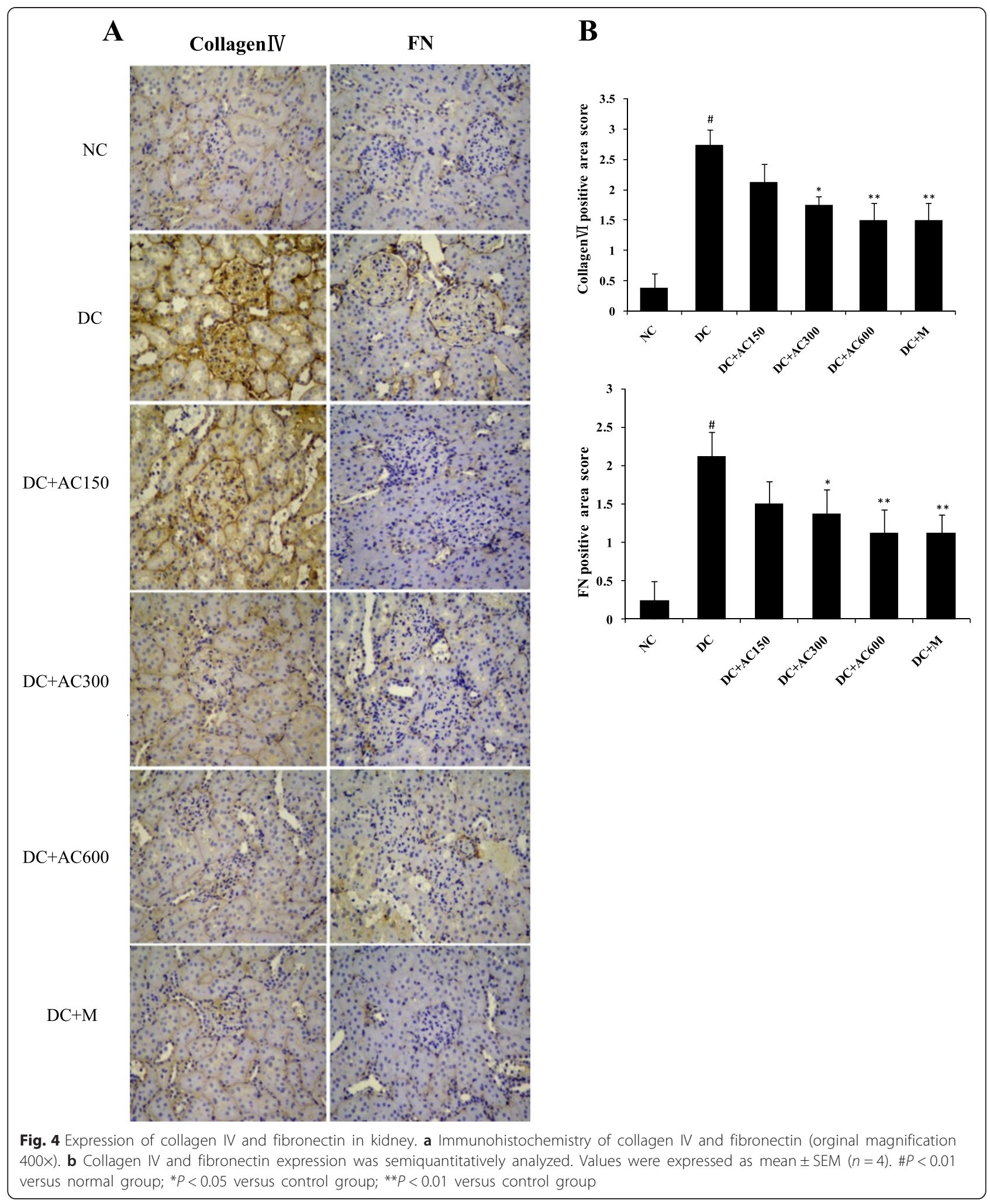

Overexpression of TGF- $\beta 1$ in diabetic kidney promotes cellular hypertrophy, stimulates ECM biosynthesis, and induces renal scarring in experimental and human DN $[27,28]$. It is well established that TGF- $\beta 1$ exerts its biological effects by activating downstream mediators of Smad2 and Smad3. The activated Smad2 and Smad3 subsequently bind to Smad4 to form the Smad complex, which translocates into the nucleus, and regulates target 


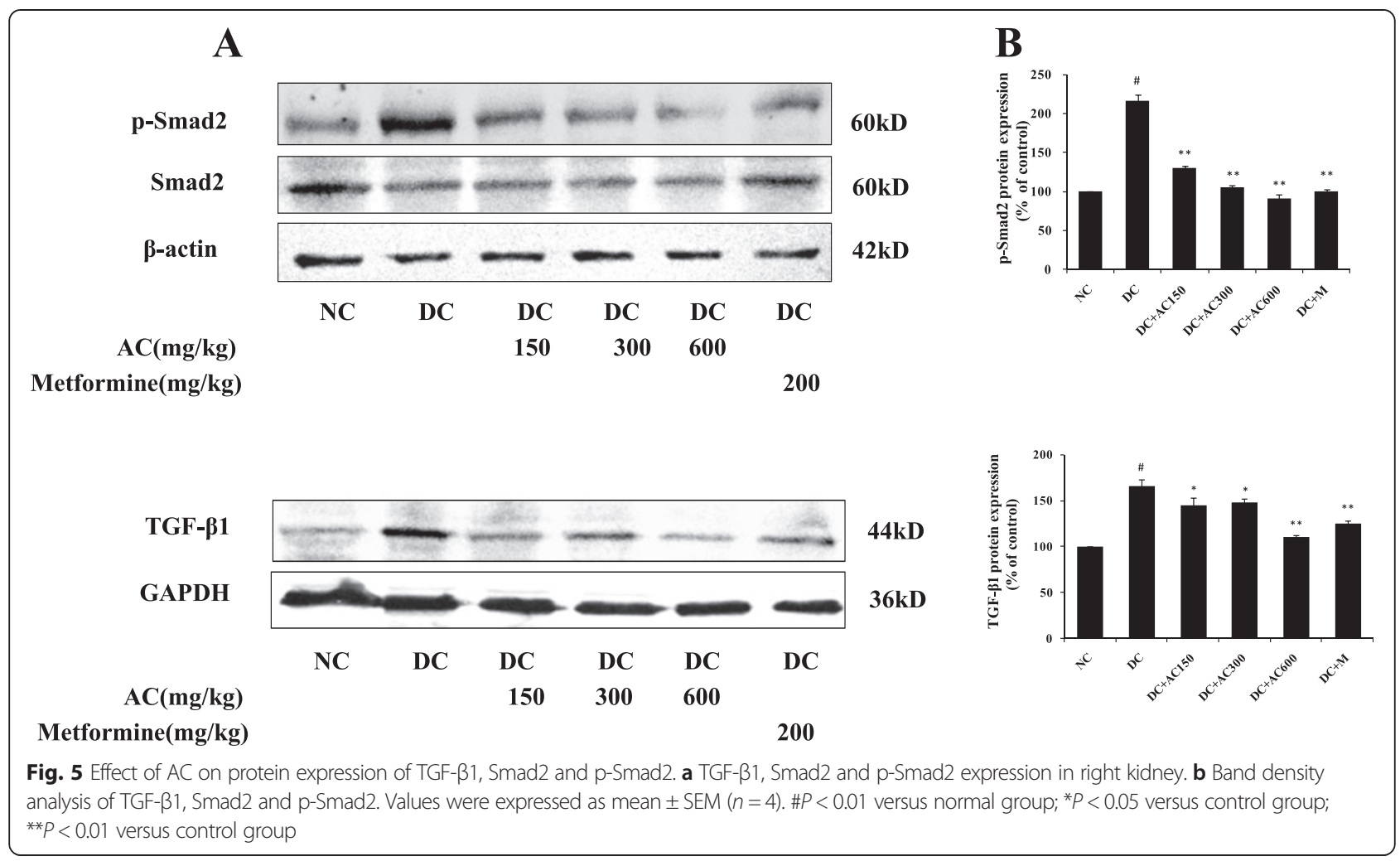

gene transcription, in conjunction with other nuclear cofactors [29-31]. Therefore, modulation of TGF- $\beta 1$ expression could be regarded as an effective therapeutic strategy for kidney diseases [29]. We showed that rats with STZ-induced diabetes had high expression of TGF$\beta 1$, major mesangial matrix expansion, and heavy collagen deposition, as well as overexpression of fibronectin and collagen IV. These results are in accordance with a previous study that showed a close relationship between overexpression of TGF- $\beta 1$ and renal morphological changes and fibrosis [28]. Diabetic rats with AC treatment showed downregulation of TGF- $\beta 1$, fibronectin and collagen IV, as well as fewer renal lesions compared with untreated diabetic rats.

AMPK acts as an energy sensor in cellular responses by raising intracellular AMP and lowering ATP [32, 33]. AMPK consists of three subunits, designated $\alpha, \beta$ and $\gamma$. The $\alpha$ subunit of AMPK contains the catalytic domain, which has two isoforms, $\alpha 1$ and $\alpha 2$. The active form of AMPK depends on the phosphorylation of threonine172 of the $\alpha$ subunit [34]. Activity of AMPK in the kidneys could be mediated by conditions of metabolic stress, such as diabetes or obesity [35]. Increasing evidence demonstrates that suppression of phosphorylation

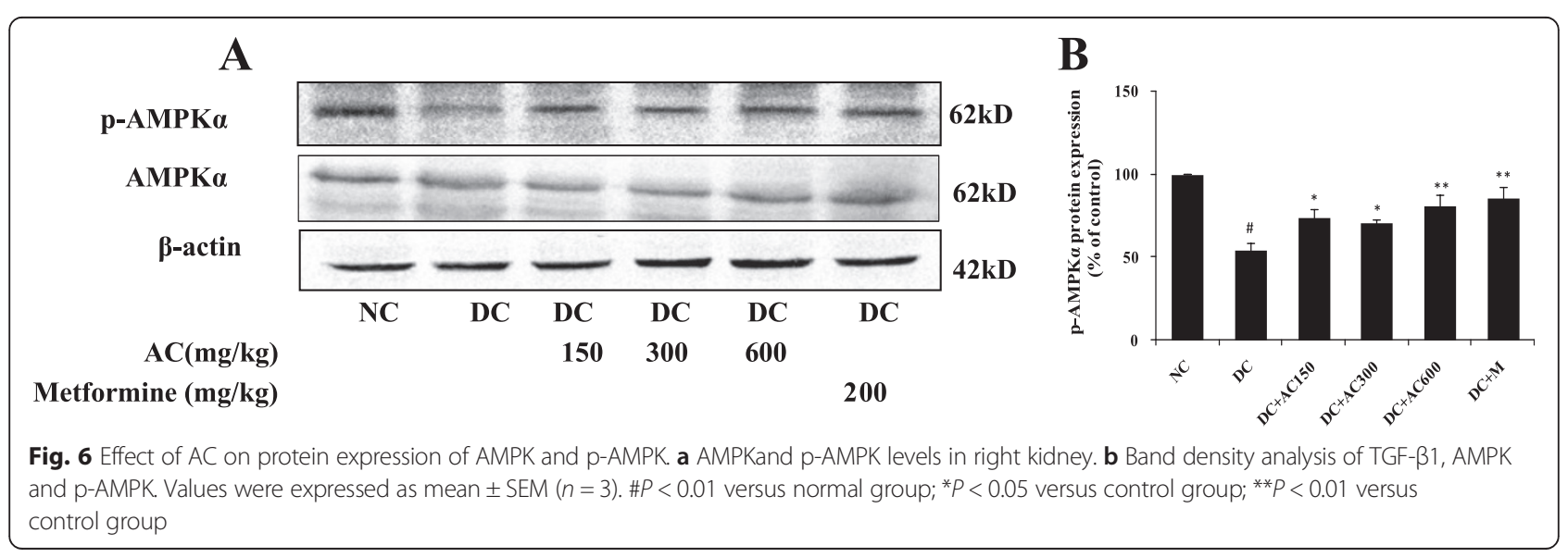


of AMPK in animal models of diabetes is involved in the pathogenesis of kidney disease through multiple pathways [36]. Recent studies have demonstrated that AMPK is closely related to renal inflammation and fibrosis [37, 38]. In vitro and in vivo experiments have confirmed that the reduction in AMPK phosphorylation could exacerbate abnormal ECM protein synthesis and renal hypertrophy by stimulating mammalian target of rapamycin activity and TGF- $\beta 1 / \operatorname{Smad} 4$ signaling pathway $[39,40]$. Furthermore, a renal antiinflammatory effect of active AMPK by suppression of proinflammatory cytokines such as MCP-1 has been identified [35, 39]. Consistently, AMPK agonists of 5Aminoimidazole-4-carboxyamide.

Ribonucleoside(AICAR) and metformin inhibit diabetesinduced renal hypertrophy and inflammation response beyond their effect on hyperglycemia [36]. Therefore, AMPK could be a target for renal protection in diabetic models. Our experiments showed a marked decrease in p-AMPK $\alpha$ expression, along with aggravation of renal inflammation and fibrosis in diabetic rats, which was consistent with previous studies. However AC and metformin treatment significantly increased p-AMPK $\alpha$ expression and ameliorated renal inflammation and fibrosis. Further investigation of the effect of $\mathrm{AC}$ on AMPK signaling pathway is needed.

\section{Conclusion}

Our results demonstrate that the renal protective effect of AC in rats with high-glucose-fat diet and STZ-induced diabetes is partially mediated by anti-hyperglycemic activity and partially by anti-inflammatory and antifibrotic activity via AMPK and the TGF- $\beta 1 / \mathrm{Smad}$ signaling pathway. However, due to the multiple compounds in AC that exert biological activities, further study is necessary to clarify the mechanism of the anti-inflammatory and antifibrotic effects of AC and its main components on DN.

\section{Competing interests}

The authors declare that they have no competing interests.

\section{Authors' contributions}

LY,carried out the experiments, data analysis and manuscipt writing. Linlin Li took part in study design. Xinxia Li and Rui Zhang carried out the ethyl acetate extraction of C. tinctoria Nutt and anlaysis of its components. Hui Li, Yujie Zhang and Jian Wang contributed to animal feeding and sample collection. Xinmin Mao designed the study, evaluated the data, and edited the manuscript for publication. All authors read and approved the final manuscript.

\section{Acknowledgements}

The present study was supported by a grant from the National Nature Science Foundation of China (No:U1303223). We gratefully acknowledge purely technical help provided by Mr Zhong Wang who is an expert in animal experiment.

\section{Author details}

${ }^{1}$ College of Traditional Chinese Medicine, Xinjiang Medical University, Xinyi Street 393, Urumuqi 830011, China. ${ }^{2}$ College of Basic Medical Sciences, Xinjiang Medical University, Xinyi Street 393, Urumuqi 830011, China. ${ }^{3}$ Center of Analysis and Test, Xinjiang Medical University, Xinyi Street 393, Urumuqi 830011, China.

Received: 22 April 2015 Accepted: 25 August 2015

Published online: 07 September 2015

\section{References}

1. Chen X, Yang W. Epidemic trend of diabetes in China: For the Xiaoren Pan Distinguished Research Award in AASD. J Diabetes Investig. 2014;5:478-81.

2. Calcutt NA, Cooper ME, Kern TS, Schmidt AM. Therapies for hyperglycaemia-induced diabetic complications: from animal models to clinical trials. Nat Rev Drug Discov. 2009;8:417-29.

3. Kanasaki K, Taduri G, Koya D. Diabetic nephropathy: the role of inflammation in fibroblast activation and kidney fibrosis. Front Endocrinol (Lausanne). 2013;4:7.

4. Soetikno V, Arozal W, Louisa M, Setiabudy R. New insight into the molecular drug target of diabetic nephropathy. Int J Endocrinol. 2014;2014:968681.

5. Lim A. Diabetic nephropathy-complications and treatment. Int J Nephrol Renovasc Dis. 2014;7:361-81.

6. Zhuo L, Zou G, Li W, Lu J, Ren W. Prevalence of diabetic nephropathy complicating non-diabetic renal disease among Chinese patients with type 2 diabetes mellitus. Eur J Med Res. 2013;18:4.

7. Ayodele OE, Alebiosu CO, Salako BL. Diabetic nephropathy-a review of the natural history, burden, risk factors and treatment. J Natl Med Assoc. 2004;96:1445-54

8. Kolset SO, Reinholt FP, Jenssen T. Diabetic nephropathy and extracellular matrix. J Histochem Cytochem. 2012;60:976-86.

9. Mason RM, Wahab NA. Extracellular matrix metabolism in diabetic nephropathy J Am Soc Nephrol. 2003;14:1358-73.

10. Jackle-Meyer I, Szukics B, Neubauer K, Metze V, Petzoldt R, Stolte H. Extracellular matrix proteins as early markers in diabetic nephropathy. Eur J Clin Chem Clin Biochem. 1995;33:211-9.

11. Ziyadeh FN. The extracellular matrix in diabetic nephropathy. Am J Kidney Dis. 1993;22:736-44.

12. Parving HH, Brenner BM, McMurray JJ, de Zeeuw D, Haffner SM, Solomon $\mathrm{SD}$, et al. Cardiorenal end points in a trial of aliskiren for type 2 diabetes. $\mathrm{N}$ Engl J Med. 2012;367:2204-13.

13. Cooper ME. Diabetes: treating diabetic nephropathy-still an unresolved issue. Nat Rev Endocrinol. 2012;8:515-6.

14. Wada J, Makino H. Inflammation and the pathogenesis of diabetic nephropathy. Clin Sci (Lond). 2013;124:139-52.

15. Garcia-Garcia PM, Getino-Melian MA, Dominguez-Pimentel V, Navarro-Gonzalez JF. Inflammation in diabetic kidney disease. World J Diabetes. 2014;5:431-43.

16. Werth S, Lehnert H, Steinhoff J. Diabetic nephropathy: Current diagnostics and treatment. Internist (Berl). 2015;56:513-9. doi:10.1007/s00108-014-3629-0.

17. Xu XX, Zhang $W$, Zhang $P$, Qi XM, Wu YG, Shen JJ. Superior renoprotective effects of the combination of breviscapine with enalapril and its mechanism in diabetic rats. Phytomedicine. 2013;20:820-7.

18. Wang W, Chen W, Yang Y, Liu T, Yang H, Xin Z. New Phenolic Compounds from Coreopsis tinctoria Nutt. and Their Antioxidant and Angiotensin I-Converting Enzyme Inhibitory Activities. J Agric Food Chem. 2014;63:200-7.

19. Li Y, Chen X, Xue J, Liu J, Chen X, Wulasihan M. Flavonoids furom Coreopsis tinctoria adjust lipid metabolism in hyperlipidemia animals by down-regulating adipose differentiation-related protein. Lipids Health Dis. 2014;13:193-211.

20. Dias T, Bronze MR, Houghton PJ, Mota-Filipe H, Paulo A. The flavonoid-rich fraction of Coreopsis tinctoria promotes glucose tolerance regain through pancreatic function recovery in streptozotocin-induced glucose-intolerant rats. J Ethnopharmacol. 2010;132:483-90.

21. Zhang Y, Shi S, Zhao M, Chai X, Tu P. Coreosides A-D, C14-polyacetylene glycosides from the capitula of Coreopsis tinctoria and its anti-inflammatory activity against COX-2. Fitoterapia. 2013;87:93-7.

22. Lu HJ, Tzeng TF, Liou SS, Da Lin S, Wu MC, Liu IM. Ruscogenin ameliorates diabetic nephropathy by its anti-inflammatory and anti-fibrotic effects in streptozotocin-induced diabetic rat. BMC Complement Altern Med. 2014;14:110-222. 
23. Tesch GH. MCP-1/CCL2: a new diagnostic marker and therapeutic target for progressive renal injury in diabetic nephropathy. Am J Physiol Renal Physiol. 2008;294:697-701.

24. Morii T, Fujita H, Narita T, Shimotomai T, Fujishima H, Yoshioka N, et al. Association of monocyte chemoattractant protein-1 with renal tubular damage in diabetic nephropathy. J Diabetes Complications. 2003;17:11-5.

25. Cheng J, Diaz Encarnacion MM, Warner GM, Gray CE, Nath KA, Grande JP. TGF-beta1 stimulates monocyte chemoattractant protein-1 expression in mesangial cells through a phosphodiesterase isoenzyme 4-dependent process. Am J Physiol Cell Physiol. 2005;289:959-70.

26. Gu HF, Ma J, Gu KT, Brismar K. Association of intercellular adhesion molecule 1 (ICAM1) with diabetes and diabetic nephropathy. Front Endocrinol (Lausanne). 2012;3:179.

27. Goldfarb S, Ziyadeh FN. TGF-beta: a crucial component of the pathogenesis of diabetic nephropathy. Trans Am Clin Climatol Assoc. 2001;112:27-32. discussion 33.

28. Chen S, Jim B, Ziyadeh FN. Diabetic nephropathy and transforming growth factor-beta: transforming our view of glomerulosclerosis and fibrosis build-up. Semin Nephrol. 2003;23:532-43.

29. Lan HY. Diverse roles of TGF-beta/Smads in renal fibrosis and inflammation. Int J Biol Sci. 2011;7:1056-67.

30. Lan HY, Chung AC. Transforming growth factor-beta and Smads. Contrib Nephrol. 2011;170:75-82.

31. Lan HY. Transforming growth factor-beta/Smad signalling in diabetic nephropathy. Clin Exp Pharmacol Physiol. 2012;39:731-8.

32. Kemp BE, Stapleton D, Campbell DJ, Chen ZP, Murthy S, Walter M, et al. AMP-activated protein kinase, super metabolic regulator. Biochem Soc Trans. 2003:31:162-8

33. Davies SP, Carling D, Hardie DG. Tissue distribution of the AMP-activated protein kinase, and lack of activation by cyclic-AMP-dependent protein kinase, studied using a specific and sensitive peptide assay. Eur J Biochem. 1989;186:123-8.

34. Hawley SA, Davison M, Woods A, Davies SP, Beri RK, Carling D, et al. Characterization of the AMP-activated protein kinase kinase from rat liver and identification of threonine 172 as the major site at which it phosphorylates AMP-activated protein kinase. J Biol Chem. 1996;271:27879-87.

35. Decleves AE, Mathew AV, Cunard R, Sharma K. AMPK mediates the initiation of kidney disease induced by a high-fat diet. J Am Soc Nephrol. 2011;22:1846-55.

36. Hallows KR, Mount PF, Pastor-Soler NM, Power DA. Role of the energy sensor AMP-activated protein kinase in renal physiology and disease. Am J Physiol Renal Physiol. 2010;298:1067-77.

37. Mishra R, Cool BL, Laderoute KR, Foretz M, Viollet B, Simonson MS. AMPactivated protein kinase inhibits transforming growth factor-beta-induced Smad3-dependent transcription and myofibroblast transdifferentiation. J Biol Chem. 2008;283:10461-9.

38. Jeong HW, Hsu KC, Lee JW, Ham M, Huh JY, Shin HJ, et al. Berberine suppresses proinflammatory responses through AMPK activation in macrophages. Am J Physiol Endocrinol Metab. 2009;296:955-64.

39. Lee MJ, Feliers D, Mariappan MM, Sataranatarajan K, Mahimainathan L, Musi $\mathrm{N}$, et al. A role for AMP-activated protein kinase in diabetes-induced renal hypertrophy. Am J Physiol Renal Physiol. 2007;292:617-27.

40. Zhao J, Miyamoto S, You YH, Sharma K. AMP-activated protein kinase (AMPK) activation inhibits nuclear translocation of Smad4 in mesangial cells and diabetic kidneys. Am J Physiol Renal Physiol 2015, 308:F1167-77. doi:10.1152/ajprenal.00234.2014

\section{Submit your next manuscript to BioMed Central and take full advantage of:}

- Convenient online submission

- Thorough peer review

- No space constraints or color figure charges

- Immediate publication on acceptance

- Inclusion in PubMed, CAS, Scopus and Google Scholar

- Research which is freely available for redistribution 